\title{
Solving Interoperability in Translational Health
}

\section{Perspectives of Students from the International Partnership in Health Informatics Education (IPHIE) 2016 Master Class}

Anne M. Turner'; Julio C. Facelli2; Monique Jaspers ${ }^{3}$; Thomas Wetter ${ }^{4}$; Daniel Pfeifer ${ }^{5}$; Laël Cranmer Gatewood ${ }^{6}$; Terry Adam ${ }^{6}$; YuChuan Li; Ming-Chin Lin; R. Scott Evans²; Anna Beukenhorst3; Hugo Johan Theodoore van Mens ${ }^{3}$; Esmée Tensen ${ }^{3}$; Christian Bock4; Laura Fendrich4; Peter Seitz; ;ulian Suleder ; Ranyah Aldekhyyel ${ }^{6}$; Kent Bridgeman ${ }^{6}$; Zhen Hu ${ }^{6}$; Aaron Sattler ${ }^{6}$; Shin-Yi Guo7; Islam Md. Mohaimenul7; Dina Nur Anggraini Ningrum; Hsin-Ru Tung7; Jiantao Bian²; Joseph M. Plasek²; Casey Rommel2; Juandalyn Burke'; Harkirat Sohi ${ }^{1}$

${ }^{1}$ Department of Biomedical Informatics and Medical Education, University of Washington;

${ }^{2}$ Department of Biomedical Informatics, University of Utah and Intermountain Healthcare;

${ }^{3}$ Amsterdam Medical Center, Amsterdam, Netherlands;

${ }^{4}$ Department of Medical Informatics, University of Heidelberg, Heidelberg, Germany;

${ }^{5}$ Department of Information Technology, Hochschule Heilbronn, Heilbronn, Germany;

${ }^{6}$ Institute for Health Informatics, University of Minnesota;

${ }^{7}$ Graduate Institute of Biomedical Informatics, College of Medical Science and Technology, Taipei Medical University, Taipei, Taiwan

\section{Keywords}

Clinical informatics, graduate education, interoperability, clinical data management, standards

\section{Summary}

Background: In the summer of 2016 an international group of biomedical and health informatics faculty and graduate students gathered for the 16th meeting of the International Partnership in Health Informatics Education (IPHIE) masterclass at the University of Utah campus in Salt Lake City, Utah. This international biomedical and health informatics workshop was created to share knowledge and explore issues in biomedical health informatics (BHI).

Objective: The goal of this paper is to summarize the discussions of biomedical and health informatics graduate students who were asked to define interoperability, and make critical observations to gather insight on how to improve biomedical education.

Methods: Students were assigned to one of four groups and asked to define interoperability and explore potential solutions to current problems of interoperability in health care.

Results: We summarize here the student reports on the importance and possible solutions to the "interoperability problem" in biomedical informatics. Reports are provided from each of the four groups of highly qualified graduate students from leading BHI programs in the US, Europe and Asia. Conclusion: International workshops such as IPHIE provide a unique opportunity for graduate student learning and knowledge sharing. BHI faculty are encouraged to incorporate into their curriculum opportunities to exercise and strengthen student critical thinking to prepare our students for solving health informatics problems in the future. 


\section{Correspondence to:}

Anne M. Turner

Department of Biomedical Informatics and Medical Education

University of Washington

Email: amturner@uw.edu

or

Julio C. Facelli

Department of Biomedical Informatics

University of Utah

421 Wakara

Salt Lake City Utah, 84108, US

Email: julio.facelli@utah.edu.
Appl Clin Inform 2017; 8: 651-659

https://doi.org/10.4338/ACl-2017-01-CR-0012

received: January 16, 2017

accepted: April 14, 2017

published: June 21, 2017

Citation: Turner AM, Facell JC, Jaspers M, Wetter T,

Pfeifer D, Gatewood LC, Adam T, Li Y-C, Lin M-C, Evans RS, Beukenhorst $A$, van Mens $H$, Tensen E, Bock C, Fendrich L, Seitz P, Suleder J, Aldekhyyel R, Bridgeman K, Hu Z, Sattler A, Guo S-Y, Mohaimenul IMd, Ningrum DNA, Tung H-R, Bian J, Plasek JM, Rommel C, Burke J, Sohi $\mathrm{H}$. Solving interoperability in translational health: perspectives of students from the International Partnership in Health Informatics Education (IPHIE) 2016 master class. Appl Clin Inform 2017; 8: 651-659 https://doi.org/10.4338/ACI-2017-01-CR-0012 Funding

Several students at the University of Utah and the University of Washington were supported by the $\mathrm{NIH}$ National Library of Medicine (NLM) Training Program in Medical Informatics (University of Washington \#T15-LM007442, Utah \#T15-LM007124). The content of this manuscript is solely the responsibility of the authors and does not necessarily represent the official views of the NIH. 


\section{Background and Significance}

The IPHIE workshop brings together an international group of faculty and students from biomedical and health informatics academic programs to share knowledge and explore solutions to health informatics problems [1-5]. The purpose of this publication is to summarize our findings on how a group of biomedical informatics students tackles a critical clinical informatics issue as recorded at the 2016 International Partnership of Health Informatics Education (IPHIE) workshop. For this exercise, we asked the students to consider the perceptions of the importance and possible solutions to the "interoperability problem" in biomedical informatics.

\subsection{Interoperability: A Central Issue in Biomedical Informatics}

The vision of an integrated healthcare system in which data from personal health, clinical health and public health systems is seamlessly and efficiently exchanged, aka interoperability, has been touted since the early 1990's when computing power and data storage became sufficient to make such systems feasible [6]. In this paper, we understand interoperability as the ability of multiple (health-related, software-based) IT systems to exchange data in order to successfully support medical research and practice by providing adequate levels of both syntactic and semantic consistency $[7,8]$.

Despite widespread adoption of electronic medical records, facilitated initially in the US by the national support provided through the HITECH Act and other similar initiatives in Europe and Asia, the vision of integrated health systems remains elusive. Consistent with this vision is the formation of health information exchanges (HIEs), i.e. networks in which health information is rapidly and accurately transferred. HIEs are commonly mentioned as a key determinant for improving health care efficiency, lowering administrative costs and improving overall health care integration $[9,10,11]$, but the impact of HIEs has been relatively minor and poorly documented [12]. Numerous systems have been developed to facilitate interoperability, including: Informatics for Integrating Biology \& the Bedside (i2b2), PCORnet, the National Patient-Centered Clinical Research Network, Strategic Health IT Advanced Research Projects (SHARP), and HL7 messaging and Fast Healthcare Interoperability Resources (FHIR), etc. $[13,14]$. These platforms contain similar components: 1) A secure infrastructure, 2) a set of common data elements and 3) standardized terminologies, which each platform implements to some extent using different base technologies.

In spite of progress being made in using such integration platforms, large scale interoperability has not been realized. Political, organizational, economic and social factors are commonly cited to explain the lack of pervasive interoperability [15]. However, semantic interoperability, defined as "the ability of two or more systems to exchange information and to use the information that has been exchanged" [16], is particularly important for the transfer of health data, and the lack of HIT interoperability has also been associated with the specific complexity of biomedical semantics. While there is a long history of standards in biomedical research and practice [17-19], use of these standards has only been partially successful. Biomedical researchers and clinical practitioners consistently report that current vocabulary standards are limiting and do not allow the full expression of biomedical concepts [20]. Ultimately, while the literature is full of references with contradictory claims on the bad actors responsible for this endemic failure [21, 22], very little consensus exists regarding the fundamental reasons for dramatically lower levels of interoperability in the health care industry when compared with many other enterprises such as travel, banking and e-commerce.

\subsection{IPHIE (International Partnership in Health Informatics Education)}

IPHIE (International Partnership in Health Informatics Education) owes its existence to an initiative of the curriculum KIK (Klinische Informatiekunde) of the University of Amsterdam, The Netherlands and its Academic Medical Centre. KIK management sought international exposure early on and first contacted the oldest European academic program in Medical Informatics cooperatively offered through Heidelberg University and - then Fachhochschule, now University of Applied Sciences - Heilbronn (Germany). The two academic institutions agreed to partner and extend their collaboration to other academic institutions. As of 2014, the following partners have joined IPHIE: The University of Minnesota (Minneapolis) in 1997, University of Utah (Salt Lake City) in 1998, The 
University for Health Informatics and Technology Tyrol (UMIT) in 2002, University of Washington in 2006, and Taipei Medical University in 2014.

A key IPHIE activity is the Master Class, in which students from partner universities meet for a special type of educational event that includes advanced lectures, discussions centered on a topic specific to biomedical informatics, and a social program to foster networking.

\section{Objectives}

We report here on the 2016 Master Class, in which participating students explored their perceptions about theoretical and practical problems precluding comprehensive interoperability in health care, as well as potential solutions to the problem. Our goal is to summarize the discussions and solutions proposed by this international group of biomedical and health informatics graduate students. We make observations on both the processes and results of this exercise to obtain insight on how to improve biomedical education by engaging diverse students in collectively addressing critical problems in the field.

\section{Methods}

The 2016 IPHIE Master Class was organized and hosted by the University of Utah (Salt Lake City, UT, USA) from July 27 -July 31, 2016. In addition to the University of Utah, participants were from the Amsterdam Medical Center, Universities of Heidelberg/Heilbronn, University of Minnesota, Taipei Medical University and University of Washington. The participants consisted of 10 faculty and 20 students who were selected based on the submission process of each of the participating universities. The main focus for the 2016 IPHIE was on student group projects in which students were asked to explore their perceptions about the challenge of achieving interoperability and brainstorm potential solutions. Students were presented with the problem and given some specific questions to guide their discussions:

- What does "Interoperability" mean?

- What are the barriers?

- What are the facilitators?

- Can it be done? If so, how? If not, why not?

To encourage students to think "outside the box", they were instructed to not be constricted by resources or current technical limitations when brainstorming new interoperability strategies, which may include both evolutionary as well as revolutionary approaches. Students were assigned to be in one of four groups and given approximately 10 hours to conceptualize the problem, identify solutions, and prepare presentations for the last day of the meeting. Each group had student representatives from different institutions and when possible a balanced mix of gender and professional backgrounds (See supplementary Appendix A Table 1). At the end of the meeting all the groups were given 30 minutes to present their projects and received feedback from faculty and students. Following the presentations and under the guidance of a designated faculty mentor, the students developed short position papers summarizing their assessment of interoperability challenges and solutions. These position papers are the basis of the results summarized in this paper. The collective critique of the student's position papers by the faculty is presented in the Discussion section of this paper.

\section{Results}

Below we provide short summaries of the students' position papers prepared by the faculty mentor assigned to the group. The full student papers represent the consensus reached independently by each group with minimal contributions from the faculty mentors. The student group position 
papers are provided in the supplementary material along with a summary of the specific critique provided by the Journal reviewers (See supplementary $>$ Appendix B).

\subsection{Group A: Smoke Signals}

Group A explained that human healthcare workers are essential for medical reasoning, as they can consider a variety of concepts (e.g. culture, lifestyle, norms and values) that are currently very difficult, if not impossible, to capture by automated reasoning. Cognitive biases may, however, affect human decision making abilities. With technological advances, the amount of medical data available to healthcare workers has grown explosively. Computers can coalesce large datasets with information from heterogeneous sources and have fewer limitations with regards to memory and capacity.

The students argue that the challenge for the present and the future is leveraging strengths of both humans and computers to achieve synergy between the two. For interoperability between humans and computers, technology is needed that enables information exchange without hindering clinical workflow. Further, social, political and organizational facilitators should be taken into account when considering interoperability issues.

For effective and efficient communication, data extraction methods are needed to alleviate healthcare workers' burden of data entry. In addition, a standard with high flexibility is required for optimal semantic interoperability to enable data exchange between all medical computer systems regardless of vendor or application. HL7 FHIR ("Fast Healthcare Interoperability Resources") is a promising resource, supporting RESTful architectures and seamless exchange of information using messages or documents. Intelligent systems predicting, preventing, and correcting possible breakdowns in the quality of information, will also improve semantic interoperability. Archetype-based, intelligent, multi-agent system techniques could use machine learning techniques to warrant reliability and quality of information. To achieve true interoperability, vendor cooperation, legally enforced or not, is vital.

Finally, it was argued by the students that users (providers, patients, administrators) should be allowed to customize software to meet their needs and complement the preferred workflow. Further advances in data visualization are needed for synthesis of the vast amounts of medical information and communicating findings back to healthcare workers.

\subsection{Group B: HICup}

Group B employed an international travel scenario to demonstrate the importance of interoperability. The Dutch Healthcare Interoperability Model (DHIM) was used to discern barriers and propose solutions. The DHIM includes five layers: Organization, Care Process, Terminology, Information Model and IT-Infrastructure. The students proposed a new component for the Personal Health Record (PWR) termed HICup "Upload your Health Information Card."

At the Organization level, students identified barriers that include devices that are not compatible with each other across countries. To solve this issue, the students suggested that the World Health Organization could coordinate development and implementation. At the Care Process level, no agreement exists about what clinical information is important or critical. Critical information could therefore be defined as diagnoses, allergies, medication, and Do Not Resuscitate (DNR) status. At the Terminology level, barriers include the different terminologies or coding system schemes used across systems. Coding the diagnoses and allergies in SNOMED CT and medications in RxNorm is a potential solution. At the Information Model level, there is no ideal information model to use; solutions proposed were to utilize HL7 FHIR, and possibly Apple Researchkit and CareKit, to draw down the healthcare data. At the IT-Infrastructure level, barriers mentioned included the need to provide off-line Internet access, use current interfaces and assure security. Solutions proposed are to store the health-related data on a chip, USB card, SD disk, or have it accessible via Bluetooth.

As it is envisioned, HICup can provide interoperable healthcare documentation. It contains only minimal critical health information, is carried by the patient and can be accessed world-wide. 


\subsection{Group C: MedBox}

Group C proposed a concept called MedBox, an electronic health record resource where the patient controls access and stores data using a MedBox compliant convention. The MedBox interoperability approach is characterized by patient control and configurable translation services based on metadata descriptions of stored data. Medbox's core element is a patient controlled, longitudinal health record that has health information from all medical encounters and also data from medical devices, fitness trackers, etc. Metaphorically, the patient holds a storage and lookup device. Actually, remote "cloud" storage and interpretation services could be used.

MedBox data contents come with a rich metadata description of the conventions applied to store the data. These conventions can be standards as well proprietary notations. For MedBox compatibility, metadata must only meet specifications of general translation services.

The students indicated that MedBox compatibility could be incentivized by making patient confirmation of a successful upload a necessary condition for re-imbursement. Interoperability, also for download from patient to provider or exchange between patients, is implied between all parties that offer the translator service compliant metadata. This approach is highly flexible by allowing new resources access anytime. It indirectly encourages standards because standard adherent resources can refer to existing standard name/version metadata rather than having to maintain their own metadata.

\subsection{Group D: Got Interoperability?}

Group D emphasized the importance of the quantifiable incentives to interoperability, such as improved quality of care, reductions in cost, and more coordinated care, reporting, and organizational operation. In order to realize these incentives and overcome barriers to interoperability, the group suggested a broad and collaborative governing organization: The Standards Management Organization (similar to the International Health Terminology Standards Development Organization IHTSDO).

The Standards Management Organization would be unique in that it is broader in scope than IHTSDO and maintains a secure repository of shareable data. The organization would provide minimum standards of interoperability. It would support institutions and providers that create biomedical data in achieving those minimum standards, while allowing them to maintain their own databases and systems so as not to disrupt workflow. Similarly, the Standards Management Organization would be involved in guiding and developing domain specific standards organizations, but would not replace them.

Finally, the organization would maintain a secure and central repository of shared data, accessible to patients, researchers, and providers at differing levels of access. The repository would facilitate care coordination, collaboration, and shared decision-making, while allowing for context-specific privacy and security measures.

\section{Discussion}

All four groups recognized the importance of interoperability, albeit two groups (B and C) focused on the importance of interoperability for a very particular use case, the International Traveler. This is an interesting use case because medical incidents for international travelers are a very small proportion of the health care provided worldwide, which is typically provided within a few miles (Km) of the patient's residence. However, this focus was likely influenced by the fact that many members of these groups were themselves from outside the U.S. and therefore were international travelers.

All groups made clear that the use of standards is critical for interoperability, but only Group A discussed how semantic interoperability is key to health information transfer and raised the question of whether it is even possible to achieve sematic interoperability. All groups appear to be very well versed in standards and quite enthusiastic about the HL7 FHIR standard. None-the-less, the enthusiasm for FHIR was not fully discussed by any group, nor were potential draw backs or limitations of this emerging standard. 
Group A provides a very interesting point of view by emphasizing the importance of interoperability between health care providers, "humans" and computer systems. They conclude that understanding how to encourage synergy between humans and computers in health care is the key to accomplishing interoperability. However, they recognized that some of the techniques needed such as speech recognition, natural language processing, and automatic semantic translation may not be sufficiently evolved in the health care domain, where "good enough" is not acceptable. Group A also discussed the use of archetypes and templates, and reflected that they may be too limited to fully express the complexity and breadth of clinical situations.

Both Groups B and C propose the use of a Personal Health Record to solve the interoperability issue, but their approaches are quite different on technology and policy. Group B appears to argue for a more top down approach, involving even the WHO and immigration authorities of multiple governments. The group did not discuss the limitations of this type of approach or discuss the lack of success of similar approaches in containing the flow of illegal drugs or terrorist suspects. Group C's approach was much more bottom up and market driven, but the students fail to recognize the lack of success of similar approaches by software giants like Google and Microsoft.

Group D's approach is quite different and argues for a classical aggregation data model in a very large (maybe nationwide or even global) data aggregation store. As with the other groups, there is very little discussion of the multiple stories (unfortunately not always published) showing plain failure and/or at least very limited success of this approach for very large health care systems.

Overall, through collaborative work in diverse groups at the IPHIE masterclass, students devised and communicated creative strategies to solve the problem of interoperability in healthcare. The experience helped them to think critically in some ways, by identifying relevant data, synthesizing results, and presenting ideas. Discussions of the student presentations and proposals during the IPHIE masterclass, including faculty comment and feedback, challenged the students to think further about the reasoning behind their solutions.

While the student position papers demonstrated that groups were well-informed about interoperability, some of the proposed solutions lacked critical thought and analysis. In future IPHIE masterclasses, to build critical thinking skills, students should be provided with the opportunity to compare and debate their approaches, and perhaps work together to reach a consensus solution. This would be a valuable way for students to reflect on faculty feedback, critique each other's ideas, and think more about the barriers to proposed solutions.

\section{Conclusions}

All four student groups identified interoperability as an important problem in medical informatics and focus on its importance in clinical settings. The students demonstrated that they are aware of the work going on with standards and distributed computing techniques. The group presentations were inventive and made uses of stories and video technologies to illustrate the problem and present their potential solutions. Each group had student representation from several participating programs and nationalities, but casual observation of students' attitudes suggested that interoperability is recognized as a critical issue regardless of nationality.

The discussion of the presentations provided an important forum for students from very diverse backgrounds to find commonalities and differences in their understanding, and propose solutions to the interoperability problem in biomedical informatics as they developed. While it is possible to argue that this experience can also be gathered by reading relevant literature and attending conferences, our observation is that the environment of the IPHIE is much more dynamic and representative of how formative ideas in biomedical informatics evolve.

From a pedagogical point of view, it appears that the latest generations, which are highly influenced by the rapid development of "news all the time", are well-informed but less trained in critical thinking than previous generations of graduate students entering our programs. Opportunities, such as IPHIE and regional or national meetings, which bring students together for code-a-thons or design competitions provide opportunities for debate, reflection and consensus [23]. The positive experience offered by IPHIE will hopefully encourage other biomedical informatics programs to 
adopt similar approaches to supplement regular didactic training with problem solving activities which will prepare our students to meet the challenges of the future.

\section{Multiple Choice Questions}

Semantic interoperability is defined as which of the following:

A) documented agreements on representation, format, definition, structuring, tagging, transmission, manipulation, use, and management of data

B) the ability of two or more systems to exchange information and use that information that is exchanged

C) the mobilization of health care information electronically across organizations within a region, community or hospital system

D)a messaging standard for exchanging clinical and administrative data between healthcare applications from various vendors, typically within an enterprise.

Answer:

Semantic interoperability is defined as B) the ability of two or more systems to exchange information and use that information that is exchanged.

The messaging standard described in answer (D) could be used to facilitate semantic interoperability, and many groups mentioned this in their papers, but the concept of semantic interoperability itself is broader. Semantic interoperability involves information exchange across organizations. Answer (A) does not talk about exchange of data. And finally, semantic interoperability deals with the ability to use information that is exchanged as opposed to merely mobilization, described in answer $(\mathrm{C})$. Therefore, $(\mathrm{B})$ is the correct answer.

\section{Clinical Relevance Statement}

Graduate students of medical informatics benefit from the diverse perspectives provided through international collaborations which foster the exchange of knowledge through problem solving of real world issues. In order to prepare students for success as clinical informatics practitioners, medical informatics training should focus on developing critical thinking skills.

\section{Conflicts of Interest}

The authors declare that they have no conflicts of interest in the research.

\section{Protection of Human and Animal Subjects}

No human or animal subjects were included in this research.

\section{Acknowledgements}

We gratefully acknowledge the generous contributions of Professor Emeritus Laël Cranmer Gatewood which made the conference possible. We would like to thank the Dieter Schwarz Foundation for supporting the IPHIE program via Heilbronn University. We also wish to thank the support of the staff and faculty of the hosting institution, the Department of Biomedical Informatics at the University of Utah, and Alyssa Bosold at the University of Washington for her help with preparation and editing of this manuscript. 


\section{References}

1. Haux R, Ammenwerth E, Ter Burg WJ, Pilz J, Jaspers MWM. An international course on strategic information management for medical informatics students: Aim, content, structure, and experiences. International Journal of Medical Informatics 2004; 73(2): 97-100.

2. Jaspers MW, Gardner RM, Gatewood LC, Haux R, Schmidt D, Wetter T. The International Partnership in Health Informatics Education. Medinfo MEDINFO 2004; 11(Pt 2): 884-888.

3. Jaspers MWM, Gardner RM, Gatewood LC, Haux R, Evans RS. An international summer school on health informatics: A collaborative effort of the Amsterdam Medical Informatics Program and IФE-the International Partnership for Health Informatics Education. International Journal of Medical Informatics 2007; 76(7): 538-546.

4. Jaspers MWM, Gardner RM, Gatewood LC, Haux R, Leven FJ, Limburg M, Ravesloot JH, Schmidt D, Wetter T. IPHIE: An International partnership in health informatics education. Studies in health technology and informatics 2000; 77: 549-553.

5. Jaspers MWM, Gardner RM, Gatewood LC, Haux R, Schmidt D, Wetter T. The international partnership for health informatics education: Lessons learned from six years of experience. Methods of information in medicine 2005; 44(1): 25-31.

6. Vest JR, Gamm LD. Health information exchange: Persistent challenges and new strategies. Journal of the American Medical Informatics Association 2010; 17(3): 288-294.

7. Hammami R, Bellaaj H, Hadj Kacem A. Interoperability for medical information systems: An overview. Health and Technology 2014; 4(3): 261-272.

8. Jiang G, Evans J, Oniki TA, Coyle JF, Bain L, Huff SM, Kush R, Chute CG. Harmonization of detailed clinical models with clinical study data standards. Methods of information in medicine 2015; 54(1): 65-74.

9. Hook JM, Pan E, Adler-Milstein J, Bu D, Walker J. The value of healthcare information exchange and interoperability in New York state. AMIA Annual Symposium proceedings / AMIA Symposium AMIA Symposium 2006: 953.

10. Hersch WR, Totten AM, McDonagh MS. Outcomes From Health Information Exchange: Systematic Review and Future Research Needs. JMIR Medical Informatics 2015; 3(4): e39.

11. Rudin RS, Motala A, Goldzweig CL, Shekelle PG. Usage and Effect of Health Information Exchange: A Systematic Review. Ann Intern Med 2014; 161:

12. Milstein-Adler J, Bates DW, Jha AK. A survey of Health Information Exchange Organizations in the United States: Implications for Meaningful Use. Annals of Internal Medicine 2011; 154(10): 666-671.

13. Ziminski TB, Algarin ADLR, Saripalle R, Demurjian S, Jackson E, editors. SMARTSync: Towards patientdriven medication reconciliation. Proceedings - 2012 IEEE International Conference on Bioinformatics and Biomedicine Workshops, BIBMW 2012; 2012.

14. Ziminski TB, Demurjian SA, Sanzi E, Agresta T. Maximizing Healthcare Delivery and Management through Technology Integration. Hershey, Pennsylvania: IGI Global; c2015. Chapter 16, Toward integrating healthcare data and systems: A study of architectural alternatives: p. 270-304.

15. Gaunt N, Roger-France F. Security of the electronic health care record--professional and ethical implications. Studies in health technology and informatics 1996; 27: 10-22.

16. Institute of Electrical and Electronics Engineers. IEEE Standard Computer Dictionary: A Compilation of IEEE Standard Computer Glossaries. IEEE Std 610. New York, New York: IEEE; 1991. 217 p.

17. Bales ME, Kukafka R, Burkhardt A, Friedman C. Qualitative assessment of the International Classification of Functioning, Disability, and Health with respect to the desiderata for controlled medical vocabularies. International Journal of Medical Informatics 2006; 75(5): 384-395.

18. Cimino JJ. Desiderata for controlled medical vocabularies in the twenty-first century. Methods of information in medicine 1998; 37(4-5): 394-403.

19. Haas SW, Travers DA. Issues in the development of a thesaurus for patients' Chief Complaints in the Emergency Department. Proceedings of the ASIST Annual Meeting 2004; 41: 411-417.

20. Gonnering RS, Ogawa GSH, Bursell SE, Horton MB. Complexity of structure in electronic medical record systems. Boca Raton, FL: CRC Press; c2014. Chapter 10, Complexity and the Human Experience: Modeling Complexity in the Humanities and Social Sciences: p. 203-219.

21. Brailer DJ. Interoperability: the key to the future health care system. Health affairs (Project Hope). 2005; Suppl. Web Exclusives.

22. Edmunds M, Peddicord D, and Frisse ME. Ten Reasons Why Interoperability Is Difficult, in: Weaver CA, Ball MJ, Kim GR, Kiel JM (Eds.), Healthcare Information Management Systems: Cases, Strategies, and Solutions, Springer International Publishing, 2016, pp 127-137.

23. Lieberman SA, Trumble JM, Smith ER. The Impact of Structured Student Debates on Critical Thinking and Informatics Skills of Second冈year Medical Students. Academic Medicine 2000; 75(10): S84-S86. 